\title{
Mathematical-computational simulation of cytoskeletal dynamics
}

\author{
Document Version \\ Submitted manuscript
}

Link to publication record in Manchester Research Explorer

\section{Citation for published version (APA):}

De Moura, C. A., Kritz, M. V., Lial, T. F., \& Prokop, A. (Accepted/In press). Mathematical-computational simulation of cytoskeletal dynamics. In Modeling and Computational Intelligence in Engineering Applications Springer Nature. http://www.springer.com/cda/content/document/cda_downloaddocument/9783319388687-c2.pdf?SGWID=0-0-451580468-p179979730

\section{Published in:}

Modeling and Computational Intelligence in Engineering Applications

\section{Citing this paper}

Please note that where the full-text provided on Manchester Research Explorer is the Author Accepted Manuscript or Proof version this may differ from the final Published version. If citing, it is advised that you check and use the publisher's definitive version.

\section{General rights}

Copyright and moral rights for the publications made accessible in the Research Explorer are retained by the authors and/or other copyright owners and it is a condition of accessing publications that users recognise and abide by the legal requirements associated with these rights.

\section{Takedown policy}

If you believe that this document breaches copyright please refer to the University of Manchester's Takedown Procedures [http://man.ac.uk/04Y6Bo] or contact uml.scholarlycommunications@manchester.ac.uk providing relevant details, so we can investigate your claim.

\section{OPEN ACCESS}




\title{
Mathematical-Computational Simulation of Cytoskeletal Dynamics
}

\author{
Carlos A. de Moura, Mauricio V. Kritz, Thiago F. Leal and Andreas Prokop
}

\begin{abstract}
Actin and microtubules are components of the cytoskeleton, and are key mediators of neuron growth and maintenance. Knowing how they are regulated enhances our understanding of neural development, ageing, degeneration and regeneration. However, biological investigation alone will not unravel the complex cytoskeletal machinery. We expect that inquiries about the cytoskeleton can be significantly enhanced if their physicochemical behavior is concealed and summarized in mathematical and computational models that can be coupled to concepts of biological regulation. Our computational modeling concerns the mechanical aspects associated with the dynamics of relatively simple, finger-like membrane protrusions called filopodia. Here we propose an alternative approach for representing the displacement of molecules and cytoplasmic fluid in the extremely narrow and long filopodia and discuss strategies to couple the particle-in-cell method with algorithms for laminar flow to model the two phases of actin dynamics: polymerization into filaments which are pulled back into the cell and compensatory G-actin drift towards its tip to supply polymerization. We use nerve cells of the fruit fly Drosophila as an effective, genetically amenable biological system to generate experimental data as the basis for the abstract models and their validation.
\end{abstract}

C. A. de Moura, Instituto de Matemática e Estatística, Universidade do Estado do Rio de Janeiro IME/UERJ, Rio de Janeiro, RJ, Brazil; e-mail: demoura@ime.uerj.br

M. V. Kritz, Laboratório Nacional de Computação Científica - LNCC, Petrópolis, RJ, Brazil; e-mail: kritz@lncc.br

T. F. Leal, Graduate Program on Mechanical Engineering, Universidade do Estado do Rio de Janeiro - UERJ; Instituto Federal do Rio de Janeiro - IFRJ, Paracambi, RJ, Brazil; e-mail: thiagofranco@ime.uerj.br

A. Prokop, Faculty of Life Sciences - FLS, University of Manchester, UK; e-mail: Andreas.Prokop@manchester.ac.uk 


\section{Introduction}

Biological phenomena rely on physical and chemical interactions. As elements of a physical phenomenon, biological components interact freely with one another: whenever in contact they exchange mass and energy. Their interactions as chemical elements are not as free, since they depend on chemical compatibilities of the interacting elements. This makes biological interactions highly complex. The elements giving cause to biological phenomena are dynamic, self-regulatory and resilient, and entities often interact through the exchange of information (signals), without being necessarily in contact or even close together.

To understand biological systems at the sub-cellular scale, we need to disentangle the organizational (functional) status of biological elements, as well as the regulatory elements, interactions and rules underlying their dynamics, from purely biochemical reactions. All biological processes are grounded on physical and chemical interactions but some of them have only sense within cells or other organisms, having no reason to spontaneously exist elsewhere.

A way to achieve this, is to have the physico-chemical dynamics well described and operationally documented as a working and consensual mathematical/computational model amenable to manipulations at higher levels, including by regulatory mechanisms induced by signaling and other stimuli. This can be achieved through the employment of mathematics and computer science to represent the behavior of complex systems occurring in life-phenomena, through structured algorithm-based simulations $[6,11,26,27,36]$. The key purpose is to represent or reproduce properties that the system under analysis displays.

Both types of observation, real experimental data and computer simulation, complement each other in several ways. Computational modeling depends on data, insights and concepts/cartoon models derived from real experimentation (in our case with biological systems). Vice versa, mathematical/computational models offer several advantages to advance the understanding of biological systems: (a) they offer inclusion of wider ranges of parameters far beyond the capacity of the human mind, (b) they can operate beyond space and time scales that can be experimentally addressed, (c) allow variables and geometries to be easily adjusted to study their impacts on the system, (d) make predictions or suggest the existence of particular interactions or structures that can then be tested experimentally, (e) provide opportunities to test assumptions and theoretical knowledge in order to refine hypotheses or suggest what might be true, (f) determine which experimental variables are most important in a system, and (g) can be used to synthesize experimental data and for testing data interpretation methods. The Nobel Prize winning physicist Kenneth Wilson proposed in the late 1980's that, for different sciences, computational modeling ought to gain the same status as theoretical analyses and laboratory experiments [62]. This enlarges the possibilities inherent in the scientific hypothesis-deductionobservation cycle and relaxes the constraints imposed by the impossibility of performing certain experiments.

We aim to model biological problems and, for this, we focus on the cytoskeleton. The cytoskeleton comprises mechano-resistant, yet highly dynamic, networks com- 
posed of filamentous protein polymers, called actin (thin filaments; diameter of 5-7 $\mathrm{nm}$ ), intermediate filaments (5-12 nm) and microtubules (thick filaments; $\sim 25 \mathrm{~nm}$ ), which support cell architecture and dynamics [21]. There is virtually no cell function that does not depend on the cytoskeleton, yet the number of essential proteins binding and regulating the cytoskeleton is surprisingly low [15, 51]. This is possible because the same cytoskeletal regulating proteins can be employed in different contexts, contributing to very distinct cytoskeletal networks and dynamics. Therefore, genetic defects of any of these components will have multiple (pleiotropic) effects, and the many human diseases caused by such defects [51] are by nature complex systemic phenomena. Furthermore, cytoskeletal dynamics are the cause and consequence of both chemical interactions as well as physical forces, and its analysis requires thinking at the interface of biochemistry and biomechanics [7].

In this chapter, we discuss a new approach to model cytoskeletal dynamics by focussing on the relatively simple context of filopodia. Filopodia are long fingerlike membrane protrusions which act as tentacle-like sensors receiving signals as well as active devices that contact other cells to convey signals. The essential architectural feature of filopodia are parallel, cross-linked bundles of actin filaments which are pulled back into the cell through disassembly processes at their base, compensated for by extensive polymerization at the very tip. This leads to a continuous treadmill-like turnover of these actin filaments. By adjusting the proportion of the polar assembly versus disassembly processes, filopodia can undergo regulated length changes. However, the high rate of polymerization at the very tip of filopodia requires uninterrupted delivery of large amounts of new building blocks which, at first sight, seems counterintuitive in these long and slender protrusions $[20,23,33,35]$. To explain this phenomenon, we propose the use of integrative models which consider catalysed actin polymerization dynamics, diffusion as well as cytoplasmic flow dynamics.

\section{The Object of our Study}

\subsection{Actin Dynamics}

Actin is the most abundant protein in eukaryotic cells [49]. Actin exists as globular actin monomers called G-actin and polar filaments called F-actin. Actin filaments are head-to-tail polymers of G-actin subunits. The minus, or pointed, end of actin filaments is relatively inert displaying slow growth in vitro. The opposite plus, or barbed, end grows much faster through exothermic polymerization both in vitro and in vivo [9].

In cells, actin filaments can be arranged into parallel bundles (e.g. in finger-like membrane protrusions called filopodia), into anti-parallel bundles (e.g. in stress fibres stretching across cells and acting as their contractile "muscle"), lattice-like networks of long filaments (e.g. in lamellar cell protrusions called lamellipodia), 
carpet-like networks of short filaments (e.g. in cortical actin underlying and structurally supporting the cell membrane), or as networks surrounding intracellular organelles [7]. The investigation of these different actin networks, in particular the flows and mechanical effects underlying plasma membrane protrusions, is an active field of experimentation and modeling, as is reviewed elsewhere [7, 15, 21, 40, 43].

As detailed in these reviews, there are three key features of actin networks (Fig. 1). Firstly, new filaments can be seeded de novo in a highly dynamic fashion through a process called nucleation, where several G-actins are catalytically oligomerized [10], from which state onwards they elongate efficiently through energy-favored polymerization. Secondly, actin filaments tend to be subjected to plus-end polymerization versus minus-end disassembly, i.e. they undergo treadmill process which translates into retrograde flow of whole actin networks if unengaged, or can become a source for force generation when linking to membrane or other cellular components [48]. Processes of polymerization and disassembly can be differentially and dynamically regulated through distinct classes of plus- and minus-end binding proteins, generating networks with different degrees of flow and constantly changing filament length. Thirdly, actin filaments can be bound one to another by a range of different cross-linking proteins or myosin motor proteins; stabilizing filaments, weaving them into different classes of networks, using them as highways for transport processes or as scaffolds to pull and generate forces [7, 40].

Of particular importance for this Chapter is the plus-end polymerization of actin. It occurs through intermolecular associations that depend on the special organization and molecular structure of the intervening monomers. Actin polymerization in cells is regulated by a number of proteins that bind G-actin and/or the actins at the plus ends of the filaments. Amongst these, Ena/VASP, formins, profilin and capping proteins are particularly important (Fig. 1). Capping proteins, such as CapZs or adducin bind and stabilise the F-actin plus end, i.e. suppress polymerization $[1,16]$.

Profilin binds and sequesters G-actin and is therefore, by default, an inhibitor of nucleation and and enhancer of depolymerization [5]. However, this role changes dramatically if formins or Ena/VASP are present and bound to the elongating plusends. These proteins bind profilin-actin with high affinity and utilize it as G-actin source to catalyze and actively promote plus-end polymerization [3, 8]. In addition, they out-compete capping proteins, and Ena/Vasp can bundle the plus ends of Factin as well as position it at the membrane [3]. Therefore, apart from the physical properties of actin and its polymerization processes, the biochemical contributions of these actin regulators need to be considered in any models aiming to describe actin dynamics.

\subsection{Filopodia}

Filopodia are relatively simple cellular compartments providing a realistic context in which to start modeling the complexity of actin network regulation in biological contexts. Filopodia are long, finger-like membrane protrusions with numerous roles 
in signaling and cell navigation (Fig. 2A) [20, 23, 33, 35]. Unlike the enormous complexity of most cellular regions with dynamically interchanging actin networks, the comparatively simple organization of filopodia offers great advantages: (1) they contain only one form of prevailing actin network consisting of parallel F-actin bundles; (2) accordingly, the number of molecular players is limited; (3) filopodial dynamics are predominantly unidimensional and (4) length changes of filopodial actin filament bundles directly translate into length changes of the entire filopodium, thus providing simple and efficient readouts for functional studies that can be carried out iteratively with modeling approaches (Fig. 2B-G) [25] .

Therefore, the regulation of filopodial dynamics appears relatively simple, essentially governed by the proteins regulating polymerization and disassembly processes. The key challenge in understanding filopodial dynamics is the high rate of polymerization at the very tip, which requires constant delivery of actin monomers through these slender and long structures. Therefore, how polymerization can be sustained within the highly challenging filopodial structure is a fascinating phenomenon which harbors key explanations for filopodial behaviors. Any models of this process will have to consider that most G-actin is likely bound to profilin which will change its diffusion properties and enormously facilitate polymerization at the plus end [3] where concentrations of G-actin can be expected to be very low. In support of this assumption, formins and Ena/VASP as active polymerizers and interactors of profilin are concentrated at filopodial tips, and deficiencies of profilin or Ena/VASP cause dramatic shortening of filopodia [25].

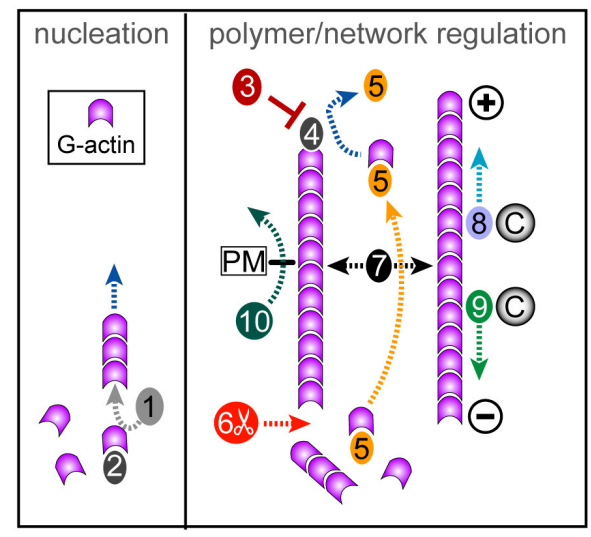

Fig. 1 Cartoon model of actin regulation. During nucleation, nucleators $(1 ;$ e.g. Arp2/3, formins) cooperate with support factors $(2 ;$ e.g. SCAR/WAVE, WASP, APC) to generate linear actin oligomers which can then undergo exothermic polymerization. Plus end polymerization is negatively regulated by capping proteins (3) that are competitively displaced by Ena/VASP (4) which, in turn, cooperates with profilin-G-Actin (5) in actin polymerization. F-actin disassembling factors (6; cofilin, myosinII) act at the minus end. Actin cross-linking factors $(7 ;$ e.g. fascin, actinin, myosin II) assemble F-actin into networks or bundles and/or exert pulling forces. Different classes of myosin motors $(8,9)$ mediate cargo $(\mathrm{C})$ transport towards the plus or minus ends of the filaments. F-actin can be post-translationally modified (PM) by specialized enzymes. 


\section{Suitable biological systems for parallel experimental approaches}

A key prerequisite for successful modeling is the existence of biological data and concepts/cartoon models. Since there is no single cellular system used in biological research which would alone provide sufficient insights and experimental data, the only feasible strategy to develop conceptual cartoon models for filopodial dynamics is to integrate mechanistic conclusions obtained from a wide range of very different cellular systems [20, 23, 33, 35]. However, this approach can be misleading since properties of filopodia may vary between animal species and cell types. Ideally, one would want a single and standardized experimental system so that data and mechanistic concepts can be reliably integrated. This would enormously facilitate the experimental validation of predictions made from mathematical modeling, especially if these predictions concern the interface between different parameters or elements.

As biological systems suitable for studying filopodia, growth cones are particularly well suited since they are rich in actin networks and reliably display filopodia as a prominent and functionally important structural feature (Fig. 2) [14, 24]. Growth cones are the motile tips of growing axons, the long and slender processes of nerve cells which form the cables that wire the nervous system. To lay these cables during development or regeneration, the growth cones at their tips navigate along specified paths, and their prominent filopodia act as sensors facilitating proper navigation [58]. Apart from the fact that growth cones display prominent filopodia with a clearly defined function, there is a good conceptual understanding of the fun-

A

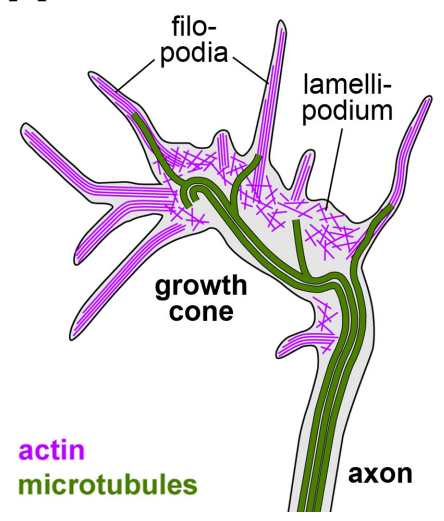

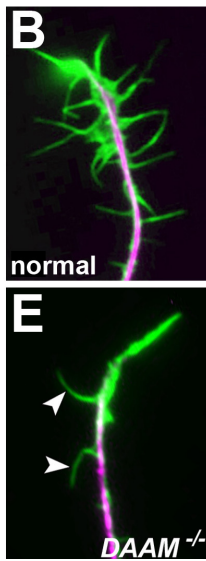

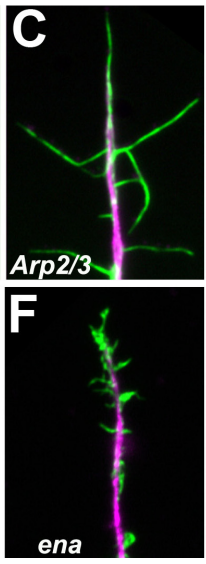

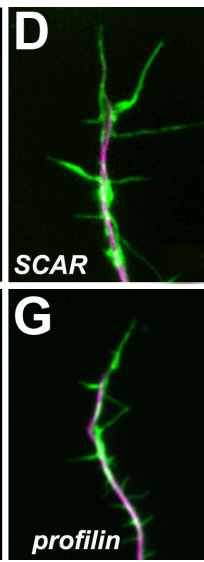

Fig. 2 Growth cone morphology. A) A growth cone showing bundles of F-actin in filopodia and lattices in lamellipodia (taken from [58]). B-G) Growth cones of fly neurons in culture lacking specific actin regulators which affect nucleation (C-E) leading to reduced filopodia number but not length, or polymerization (F, G) primarily affecting filopodial length (modified from [25]) 
damental roles that actin plays in growth cones, and their actin machinery is well investigated in this context [24, 44].

More recently, growth cones of the fruit fly Drosophila have been established as powerful models accessible to detailed studies of the cytoskeleton and systematic genetic dissections of its various regulators [51, 52, 57, 58]. For example, a systematic study used filopodial number and length as a simple readout to understand the systemic contributions and functional interfaces of seven different actin-binding regulators, which included formins, Ena/VASP, profilin and capping proteins (Fig. 2B-G). This work demonstrated how Drosophila growth cones can be used to deduce cartoon models of filopodial dynamics [25, 53]. Given the relative speed and ease with which experiments can be carried out in Drosophila neurons, this system is ideal for partnering up with the mathematical/computational modeling of filopodial dynamics [51].

\section{Modelling Filopodia}

\subsection{Previous Models}

Since the end of the 1950's, researchers have been developing models to quantitatively validate experimental results from their laboratory experiments. Very early on, Oosawa and colleagues found that actin polymerization processes were dependent of G-actin concentration [41]. In another early seminal study, Wegner mathematically described actin treadmilling [61]. A further good example is the ordinary differential equation model by Bindschadler and colleagues which explicitly accounts for nucleotide-dependent actin polymerization and depolymerization [4].

At about the same time, taking advantage of technological advance and detailed experimentally measured data, Vavylonis and colleagues built a quantitative discrete model of actin polymerization [60]. This model could be validated by new in vitro experiments using reflection fluorescence microscopy [22]. Mechanisms of actin polymerization have been studied including the interactions between actins and actin binding proteins (ABP's) and the rate constants for each step leading to quantitative models of polymerization [49].

Another important model that elucidated thinking about how actin polymerization can dynamically control cell shape and motility was the Brownian ratchet model by Peskin and colleagues [46] which aims to explain how chemical reactions generate protrusive forces by rectifying Brownian motion. The ratchet mechanism is the intercalation of monomers between the barrier (e.g. membrane) and the polymer tip. A particle diffuses in one dimension ahead of a growing bundle of filaments, executing a continuous random walk in a constant force field. As a result, it analyzes polymerization velocity as a function of the load force.

Actin dynamics in motile cells have also been the subject of many modeling approaches. For example, the model by Mogilner and Keshet showed how membrane 
speed depends on actin monomer concentration and cycle (ATP hydrolysis, F-actin treadmilling), reactions with actin-binding proteins and barbed end polymerization at the leading edge of a cell [39]. Dawes and colleagues investigated the spatial distribution of actin filaments and their barbed ends, and the interplay between filament branching, growth, and decay at the leading edge [12]. Others investigated the mechanisms of pushing and pulling by actin and microtubules [37], the relations between protrusions, monomer concentration and stiffness of the filament bundles [38], or anterograde flow of cytoplasm to provide sufficient material for the extension of protrusions, such as lamellipodia [29].

As these examples illustrate, the modeling of actin in biological contexts has to include different levels of resolution, such as structural, physical and chemical properties of actin filaments and protein complexes that govern F-actin dynamics [7].

With regard to modeling filopodia, several previous attempts should be mentioned. For example, Lan and Papoian used stochastic simulations of filopodial dynamics, discretizing space into compartments and simulating protein diffusion along the filopodium as a random walk [30]. Erban and colleagues modeled the problem of G-actin delivery to the filopodial tip based on diffusion, 3D stochastic models, and comparing compartimental and molecular models as strategies to simulate actin dynamics [18]. An impressively comprehensive model was published by Mogilner and Rubinstein who included aspects such as physical properties, crosslinkage and numbers of filaments, membrane resistance as well as the G-actin diffusion coefficient [38]. Another recent model by Zhuravlev and colleagues proposed that a key limiting factors of filopodial length is diffusional transport of G-actin monomers to the polymerizing barbed ends, and they investigated potential roles of active motor-driven transport of G-actin [64]. They concluded that "a naive design of molecular-motor-based active transport would almost always be inefficient - an intricately organized kinetic scheme, with finely tuned rate constants, is required to achieve high-flux transport".

\subsection{Can Diffusion Alone Explain G-actin Delivery to the Filopodial Tip?}

We first explored whether, and under which conditions, diffusion might be sufficient to supply barbed-end actin polymerization processes in filopodia considering the work by others who investigated actin diffusion as a key mechanism in filopodia. For reasons of simplicity, our calculations do not consider filopodial elongation, but analyze conditions where retrograde flow and polymerization are in balance, thus maintaining filopodial shape, length, retrograde flow in a steady state. For our calculations we used the parameter values indicated in bold text in Table I. 
Table I Parameters and data regarding filopodia and actin. Values selected for this work are shown in bold.

\begin{tabular}{|c|c|c|c|}
\hline Notation & Meaning & Value & References \\
\hline$L$ & $\begin{array}{l}\text { Filopodial } \\
\text { length }\end{array}$ & $\begin{array}{c}24-55 \mu \mathrm{m} \\
0.03-0.15 \mu \mathrm{m} \\
1-10 \mu \mathrm{m} \\
10-20 \mu \mathrm{m} \\
3-10 \mu \mathrm{m} \\
\text { Here: } \mathbf{1}-\mathbf{3 0} \mu \mathbf{m}\end{array}$ & \begin{tabular}{|l|}
{$[2]$} \\
{$[37]$} \\
{$[46]$} \\
{$[54]$} \\
{$[57]$}
\end{tabular} \\
\hline$N$ & $\begin{array}{l}\text { Number of filaments } \\
\text { in the filopodial bun- } \\
\text { dle }\end{array}$ & $10-30$ & $\begin{array}{l}\text { References } \\
\text { in [38] }\end{array}$ \\
\hline $\mathrm{C}_{0}$ & $\begin{array}{llr}\text { G-actin } & \text { concentra- } \\
\text { tion an } & \text { filopodial } \\
\text { base } & & \\
\end{array}$ & $10 \mu M$ & [38] \\
\hline$k_{\text {on }}$ & $\begin{array}{l}\text { Polymerization } \\
\text { rate }\end{array}$ & $\begin{array}{c}10 \mu M^{-1} s^{-1} \\
11.6 \mu M^{-1} s^{-1}\end{array}$ & $\begin{array}{l}{[38]} \\
{[65]}\end{array}$ \\
\hline$N_{0}$ & $\begin{array}{l}\text { Number of filaments } \\
\text { to support protrusion }\end{array}$ & 13 & {$[38]$} \\
\hline$\eta$ & $\begin{array}{l}\text { Unit conversion fac- } \\
\text { tor }\end{array}$ & 20 & [38] \\
\hline$\delta$ & $\begin{array}{l}\text { Half-size of actin } \\
\text { monomer }\end{array}$ & $2.7 \mathrm{~nm}$ & $\begin{array}{l}\text { References } \\
\text { in [46] }\end{array}$ \\
\hline $\bar{D}$ & \begin{tabular}{|l|}
$\begin{array}{l}\text { G-actin diffusion } \\
\text { coefficient }\end{array}$ \\
\end{tabular} & $\begin{array}{l}4 \mu \mathrm{m}^{2} / \mathrm{s} \\
\mathbf{5} \mu \mathbf{m}^{2} / \mathbf{s} \\
\end{array}$ & \begin{tabular}{|l}
$37]$ \\
{$[34]$} \\
\end{tabular} \\
\hline$V_{\text {ret }}$ & Retrograde flow rate & $\begin{array}{c}\mathbf{7 0} \mathbf{~ n m} / \mathbf{s} \\
30-80 \mathrm{~nm} / \mathrm{s}\end{array}$ & \begin{tabular}{|l}
{$[38]$} \\
{$[57]$}
\end{tabular} \\
\hline
\end{tabular}

In short filopodia of $1-2 \mu m$, the concentration of free actin at the filament base is still sufficient to supply enough polymerization for filopodial extension to occur [38]. This is in agreement with (1) for estimating the approximate time required for a particle to diffuse over a given distance $x$, in an environment where its diffusion coefficient is $D$

$$
t \approx \frac{x^{2}}{q_{i} D}
$$

In (1), $q_{i}$ is 2,4 or 6 depending on the number of dimensions ( $i=1,2$ or 3 ) [28]. Considering a linear displacement $\left(q_{i}=2\right)$ and a diffusion coefficient of $5 \mu \mathrm{m}^{2} / \mathrm{s}$, a G-actin travels $3.16 \mu \mathrm{m} / \mathrm{s}$.

For filaments longer than a few micrometers, we can analyze concentration of free actin according to expression (2) mentioned in [38]:

$$
C(x)=C_{0}-\frac{C_{0} x}{\left(L(t)+\left(D \eta e^{N_{0} / N}\right) /\left(k_{o n} N\right)\right.}
$$


In (2), $L(t)$ indicates filopodial length as a function of time (i.e. considering filopodial elongation or shrinkage). Our work does not consider elongation, so we assume $L(t)=L$ constant. Furthermore, we chose the filopodial length interval to cover for a wide range of natural filopodia (Table I), although values between $10-20 \mu \mathrm{m}$ are more frequently observed [7]. Function $C(x)$ is the G-actin concentration at a given distance between the filopodial base $(x=0)$ and the filopodial tip $(x=L)$. Therefore, the free actin concentration at the tip of the filopodium is given by the function expressed in

$$
C=C\left(L, N, k_{o n}\right)=C_{0}-\frac{C_{0} L}{L+\left(D \eta e^{N_{0} / N}\right) /\left(k_{o n} N\right)}
$$

This value allows for calculating how many actins reach the polymerization point $N_{p}$ in scenarios generated by varying $L, N$ and $k_{o n}$. The chosen values are in the ranges reported in Table I.

The key question is whether diffusion is sufficient to sustain polymerization in order to balance the actin-bundle retrograde flow $\left(V_{\text {ret }}=70 \mathrm{~nm} / \mathrm{s}\right.$ in Table I). To achieve this velocity, a polymerization frequency of 30 actins $/ s$ per filament in the bundle is required when considering that every new actin elongates a filament by $\approx 2.7 \mathrm{~nm}$ (Table I). Therefore, in a bundle with $N$ filaments, $N_{\text {ret }}=30 \mathrm{~N}$ actins should be polymerized per second. This will be compared with the number of actins obtained from (3), to check if diffusion is enough to sustain F-actin retrograde flow.

At a G-actin concentration of $10 \mu M$, the polymerization rate per actin filament was reported to be $0.3 \mu \mathrm{m} / \mathrm{s}$, which means that 110 actins are polymerized in a single filament per second [47]. For a bundle with $N$ filaments, the number of polymerizing actins $N_{p}$ would be $110 N / s$. By integrating this with expression (3, we have:

$$
N P=110 N C
$$

as the number of actin polymerized at a given concentration $C=C\left(L, N, k_{o n}\right)$. The calculations were performed by the following routine:

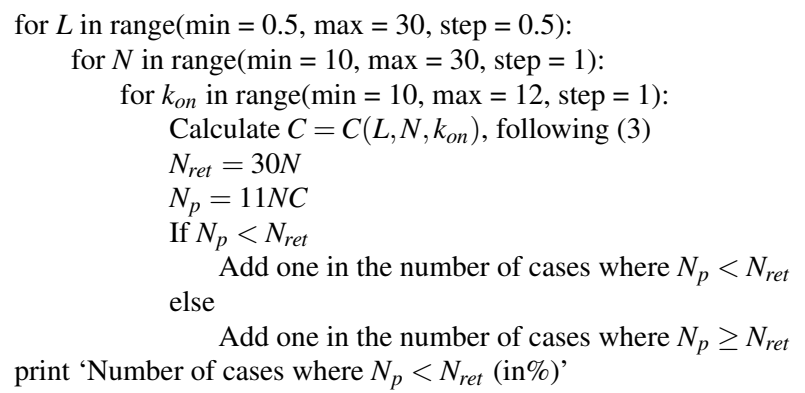

Here $N_{p}=11 N C$, because the number of polymerized actins is directly proportional to the concentration of monomers. At a concentration of $10 \mu M, 110$ actins are polymerized per second on each filament, providing 11 actins $/ s$ at $1 \mu M$. The expression (3) yields $C \rightarrow C_{0}$ when $L \rightarrow 0$, ensuring that $N_{p}=110 N$ for a concentration close to that at the base of filopodia. 
Using this algorithm in combination with the values given in Table I, we obtain that of all cases the polymerization does not supply retrograde flow in $90.32 \%$. In comparison, when setting the filopodial length interval to $L \in[0.5,2] \mu m$, diffusion seems ineffective in just $15 \%$ of cases. This result suggests that diffusion alone is not sufficient in many scenarios, especially when reaching filopodial lengths beyond $1.5 \mu M$, and when $N$ is greater than 21 .

Other reports agree with our view. For example, Monte Carlo simulations were used to investigate G-actin translocation during protrusion of the leading edge leading to the conclusion that diffusion alone was insufficient [63]. The use of compartmental and molecular stochastic models to study actin motion by diffusion concluded that filopodia would reach a steady state length of as little as $\approx 1 \mu \mathrm{m}$ because the transport flux of G-actin monomers continuously diminishes as the tube becomes longer [18]. Also work on filopodia-like acrosomal processes of sperm found that the kinetics of diffusion-limited actin polymerization were not sufficiently rapid to account for the observed acrosomal elongation dynamics [45].

\subsection{An Alternative Approach}

The above calculations show that diffusion alone is, in general, not enough to supply the amount of G-actin needed to sustain the observed rates of polymerization and backflow. As one compensatory mechanism, Zhuravlev and colleagues proposed active motor-driven transport of G-actin [64], but there is hardly any experimental proof for this hypothesis. Therefore, we propose in the sequel a modelling approach which integrates a number of very different physical and chemical processes within filopodia (Fig. 3A): diffusion of actin building blocks, support-factor aided polymerization at the filament tips, as well as cytoplasmic flow driven by the forces caused by the displacement of actin filaments towards the filopodial base.

The idea of protein-mediated facilitation of polymerization was already explained in Section 2.1. Here, we briefly explain the concept of cytoplasmic flow. Thus, F-actin filaments at the core of the filopodium are constantly flowing backwards from the tip towards the cell through disassembly processes occurring within the cell (Fig. 3A). This causes loss of volume in the filopodial tip which comprises the actin filaments, their hydration, other attached proteins and perhaps even cytoplasm trapped inbetween the tightly packed actin filaments. If this volume loss is combined with sufficiently resistant membrane structure to prevent membrane collapse (open arrow in Fig. 3C), this would generate a negative pressure that needs to be compensated for by incoming cytoplasm to prevent cavitation.

Indeed filopodia seem to display such membrane rigidity provided by a class of proteins containing BAR (Bin-Amphiphysin-Rvs) domains (Fig. 3A) [32, 55, 56]. Given therefore a relatively stable shape of filopodia, the "outflow" of volume can be expected to drive a compensatory influx in the space between actin bundle and cell membrane towards the tip of the filopodium (Fig. 3C). The inflowing volume should be a mixture of cytosol (colloidal water) and G-actin molecules where the latter are 
still free to diffuse (Fig. 3D). At the tip of the filament the mixture-flow bends in the direction of the polymerization points, guided by the molecular organization around it, where the diffused G-actins are re-arranged through polymerization into the backflowing filament bundle. The bending flow might even produce outward forces in the membrane that could further help sustain its form.

\section{A Model for Filopodial Dynamics}

\subsection{Principal Thoughts about Strategic Choices}

Three processes are central in the above description: diffusion, guided polymerization and cytoplasmic flow. With polymerization being a chemically reactive process, we have a diffusion-reaction system with advection. In principle, there are several

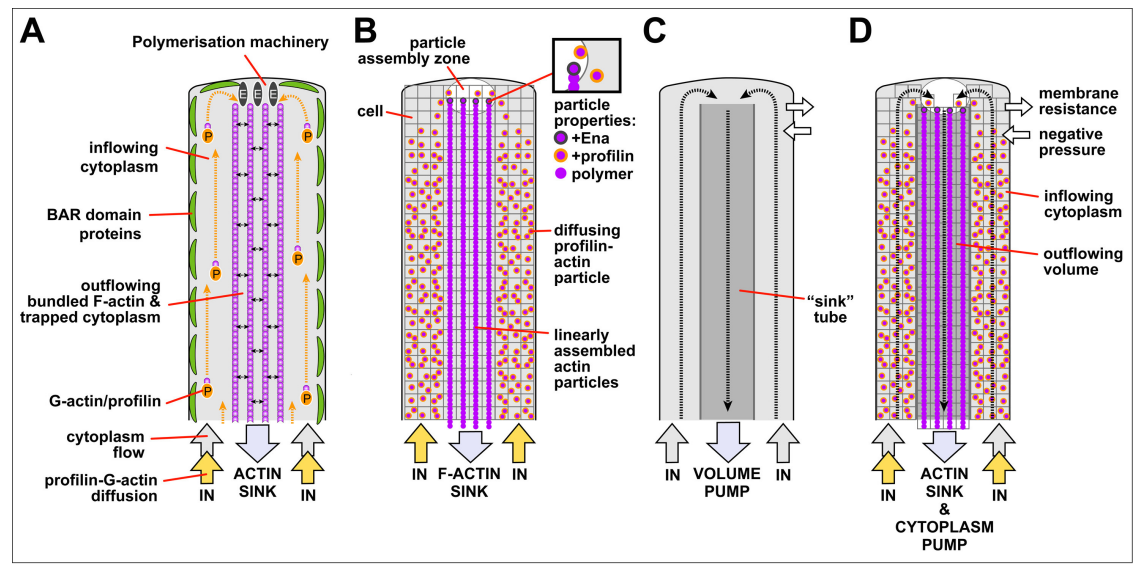

Fig. 3 Cartoon models of filopodial dynamics. A) Biological model: in cells, actin bundles are pulled out of filopodia from their minus ends (ACTIN SINK) which is compensated through Ena/VASP-mediated (E) plus end polymerization requiring supply (yellow arrows) with profilin (P)-bound G-actin (same symbols as explained in Fig. 1). Filopodial membranes are stabilised by BAR domain proteins. B-D) Mathematical/computational models: B) A model of diffusion and polymerization: the filopodium is subdivided into a coordinate system within which actin particles (magenta dots) undergo Brownian movement, diffusion and assembly processes; to reflect contributions by regulatory proteins, actin particles are equipped with profilin- or Ena/VASP-bound properties (orange or brown circles; see close-up top right). C) Flow model: filopodia are represented by a tube-in-tube constellation where volume (actin filaments with potentially trapped cytoplasm) flows out of filopodia, driven by a pump at the base of the inner tube, causing compensatory inflow of cytoplasm. D) Combinatorial model: the coordinate system becomes dynamic reflecting the circulating cytoplasm flow from $\mathbf{C}$; actin particles with different properties (as in $\mathbf{B}$ ) can diffuse and polymerize within this dynamic context, thus combining cytoplasmic flow, diffusion and regulator-mediated polymerization. 
standard models from several mathematical disciplines which can describe such processes.

However, the mathematical modeling approaches to implement advection dynamics are very different from, and complementary to, those required by reactiondiffusion. Despite its hydro-dynamical elegance, the alternative approach above, that suggests a re-organisation of particles within a flowing cytosol, cannot arise under the commonly used diffusion perspective because cytosol displacements are disregarded and only diffusive movements are represented. A new starting point is needed to suggest the models and observations needed to test it.

Furthermore, setting the standard equations for reaction, diffusion and advection per se, down as a model for filopodial dynamics is challenging, because they were developed based on very basic principles of homogeneity, uniformity, and isotropy, and the substances that react or drift are supposed to be present at any point of the domain. However, filopodia are anything else than isotropic or uniform. Different processes are predominant in very distinct regions of the filopodia and, thus, different equations should be used in distinct subregions of the model's domain. For example, the standard equations allow, indeed enforce, actin reactions to take place anywhere in the filopodium and consider actin molecules as uniformly charged spheres, hampering the representation of what we know about protein-aided polymerization reactions which occur only at the plus-end of each filament. Furthermore, filopodia are bounded by (1) the moving cell membrane and (2) a rather unspecified surface at the bottom of the protrusion, and (3) further sub-domains are formed by the dynamic flow of distinct molecule classes: the backflowing actin bundles versus the (laminar) advection outside them. Therefore, the geometrical boundaries of each sub-domain cannot be determined in advance, as they result from molecular dynamics. Taking into consideration that we need to discretise the equations to perform simulations and numerical experiments, and that we are dealing at the very validity limit of continuous flow models [31], our chance of getting close to helpful and interesting solutions in this way is not very likely.

Based on these considerations, we prefer therefore computational models based on both mathematical and biological knowledge and tailored to what is known to happen in each subdomain of a filopodium. We consider here the combined use of two computational methods. First, the finite volume discretization with moving elements method, based on standard models of laminar flow and discretization methods which can track changes in the sub-domain geometry [13]. Second, the particle-incell method (PIC) relating to reaction-diffusion equations. This method can be used to model stochastic processes and simultaneously represent the rigid motion of the F-actin filament bundle, simply by considering transitions to be deterministic and remaining in a direction given by the central axis of the cylindrical portion of the filopodium. 


\subsection{Modelling F-actin Polymerization and Diffusion}

The PIC method we chose, is a numerical technique that deals with problems where a fluid can be seen as particles scattered in a field described by a mesh, cf. [19, 50]. Every grid cell has a sufficiently large quantity of particles interacting with each other, carrying information about its own position and physico-chemical properties. PIC exhibits a widespread usage in computer simulation of plasma physics, gases or other fluids composed of particles with high kinetic energy. It seems an efficient strategy to simulate the two-phase mixture here described.

Filopodia contain a collection of interacting particles and the cytosol. By particles we mean G-actin, F-actin and actin binding proteins - ABP's, i.e. the key factors that interact and regulate the entire system. They are placed over a background field formed by the cytosol fluid, or cytoplasm. Our model domain is an environment that involves a multi-scale fluid observation, in order to consider relevant, microscopic, physical phenomena that bear influence on macroscopic properties of fluids.

In our chosen PIC model, each particle's position is updated in response to system stimuli, such as fluid dragging, collision with other particles or chemical reactions. For example, polymerization processes, are triggered by the proximity of G-actins with the tip of F-actin bundles to which they bind. In this regime, distance can be used as a priority-parameter to decide which molecule polymerizes (Fig. 4).

In addition, the position-tracking of particles permits to measure deformations through observation of displacements of F-actin position (Fig. 5). This information will be relevant in future analyses, for example when incorporating forces, F-actin buckling and membrane resistance.
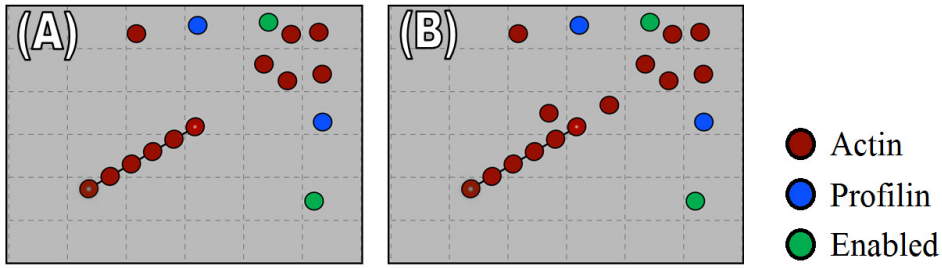

Fig. 4 Schemes to represent possibility of particle interactions in our PIC simulations. (A) Several particles can be moving within same tiles (B) If a G-actin is in the same tile of polymerization point, it can be polymerized

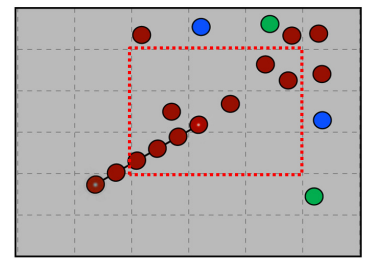

Fig. 5 Deformation of the filament measured through changes in particle positions 
In our preliminary simulations, particles move in Brownian motion through a 2D domain, and we observe G-actin concentration depletion in the area around a polymerization point (red dot in Fig. 6). This is an expected behavior since G-actins are consumed by polymerization and Brownian motion of other particles is not efficient enough to fill the vacant spaces.

\subsection{Modelling Cytosolic Flow as a Means to Drive Passive Transport of G-actin}

In order to investigate how G-actins are delivered to the polymerization points, we must consider physical properties of cytosol and fluctuations in the concentration of G-actins and other molecules throughout the domain. Therewith, we analyze cellular compensatory mechanisms for replacing the molecules consumed by polymerization, subject to the principle of mass conservation. Cytosol has a colloidal-water constitution which, in first approximation, leads to considering it as an incompressible and viscous fluid and modeling its motion by Navier-Stokes equations for incompressible fluid flow [31],

$$
\begin{array}{r}
\rho\left(\frac{\partial \mathbf{U}}{\partial t}+(\mathbf{U} \cdot \nabla) \mathbf{U}\right)=-\nabla p+\mu \mathbf{U}+\mu \nabla^{2} \mathbf{U}+F \\
\frac{\partial \rho}{\partial t}+\nabla \cdot(\rho \mathbf{U})=F_{m}
\end{array}
$$

Here $\mathbf{U}$ stands for the velocity vector, $\rho$ for the fluid density, $\mu$ for the dynamic viscosity, $p$ for the hydrostatic pressure, $F$ for the source of movement and finally $F_{m}$ for the source of mass. Considering filopodia as a system where fluid velocities are low, with high viscosity and very small length-scales, Navier-Stokes above equations (5-6) can be simplified and written as:

$$
\nabla p-\mu \nabla^{2} \mathbf{U}=F
$$

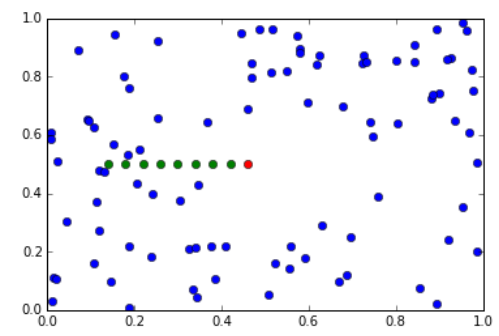

Fig. 6 Particles (G-actins, blue) moving in Brownian motion, F-actins in green with the polymerization point (red dot) from where these filaments elongate. Note the decreasing G-actin concentration around the polymerization point. 


$$
\nabla \mathbf{U}=F_{m}
$$

taking into account that $\rho=$ constant, due to fluid incompressibility. Flows with these characteristics are called Stokes flows and equations (7-8) are known as Stokes equations.

At this point, the fluid can be analyzed through a macroscopic approach, i.e., in a laminar flow with a certain concentration of G-actins in suspension, since the latter are supposed to be passively transported by the cytosol and do not affect its flow. This scenario is based on assumptions described in previous sections: the volume removed from the filopodia tip induces a hydrostatic pressure, thus leading to the cytosol displacement.

The conditions and processes we seek to investigate with the tools under development include the rules underlying G-actin motion, hydrostatic pressure and membrane resistance. Aiming to enforce mass conservation inside the filopodia, which is a critical aspect of our approach, we have decided to discretize the model flow equations with the finite volume method. Notably, PIC can be coupled with finite volume laminar flow giving an integrated two-phase representation of an entire system as a dynamic fluid, to study the reorganization of the actin-phase into filaments at molecular scales. Within a neighbourhood of the polymerization points, the two methods merge by superposition: the PIC method determines the movement of G- and Factins relative to the volume elements, and the finite volume algorithm drives the displacement and deformation of these volume elements. Volume elements trapped within the F-actin filament bundle move then with the filaments.

The domain of our model mimics filopodia interior and will initially be twodimensional for implementation tests. The scheme in Fig. 7 represents a filopodium and a bundle of actin filaments inside, where red arrow means retrograde flow. The cytoplasmic fluid is pumped from lamelipodia in $x=0$, for $y \in\left[0, \delta_{1}\right) \cup\left(\delta_{2}, \delta\right]$. Filopodium is also bounded by the membrane at $x=L$. Actin polymerization occurs at $x=L_{0}$ where $y \in\left[\delta_{1}, \delta_{2}\right]$. This region is the inset of a tube that represents F-actin filament bundle and drive actins out of filopodia, acting as a sink.

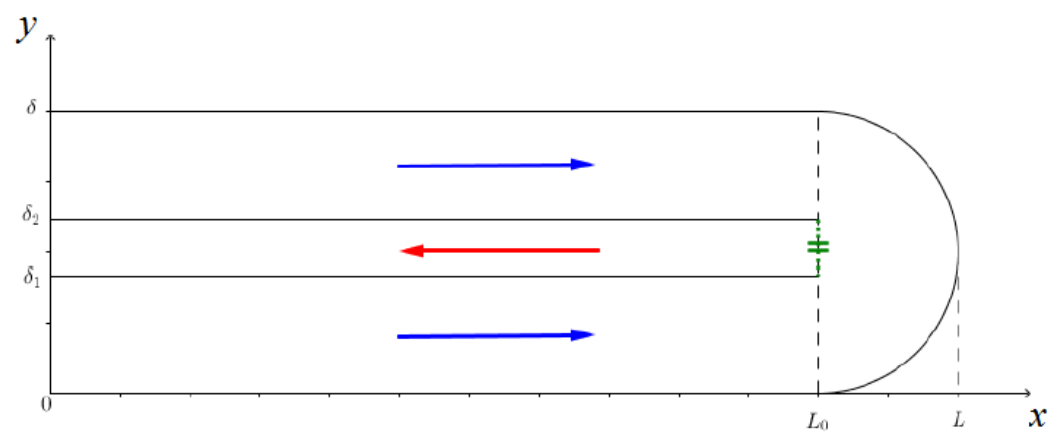

Fig. 7 Filopodia fluid motion scheme 


\subsection{Model Validation}

Model validation is a long-term project which relies on the confidence we gain from solving questions and explaining biological measurements with our model. Nevertheless, there are series of immediate experiments that can be undertaken to check both the model's conceptual soundness and its implementation.

We have seen in Section 5.2 that the PIC model represents well the diffusion of G-actins, the formation of filaments, and their almost rigid-like motion along a line. Computational models for laminar flows based on finite volumes are well known, as well as their validation. The steps below address the coupling of both algorithms and the representation of characteristics relevant for biological enquiries and the special filopodia geometry.

First, we need to verify basic aspects: mass conservation, soundness of the flow bending at the tip, coupling of advective currents with the polymerization points, and the return of G-actins by the cytosolic inward flow. Furthermore, we need to verify if the superimposition of both models retains properties relevant for the biological problem: does it (1) provide enough actins at the polymerization points? (2) reduce the flow when polymerization stops? (3) represent elongation and retraction of the filopodia?, and so on.

Moreover, the model can be used to perform tests that provide data supporting the approach proposed, like those suggested in Fig. 8. The results of numerical (virtual) experiments of this type provide data in favour or against the proposed approach.

\section{Conclusions}

This chapter presents a novel approach to the description of the flow of matter inside filopodia, adding advection of the cytosol to the diffusion of molecules suspended or dissolved within it. Proposing this advection is justified by the biological knowledge that volume (actin, attached proteins, cytosol) is withdrawn from the filopodia, providing a pump mechanism for compensatory inflow. This research is in its infancy and a lot of open questions lay ahead, besides those related to model validation. These open questions include: (1) relations between the number of filaments in the bundle and several aspects, like flow velocity, G-actin concentration, elongation-retraction of the protrusion, etc; (2) the mechanical effects or the flow over the membrane throughout the filopodia; and (3) how the outward movement of the filament bundle affects the laminar inward flow. We are working towards joining the PIC and the finite volume algorithms and hope to be addressing these intriguing questions in the near future and couple them up with imaging experiments in Drosophila growth cones. However, our ultimate goal is to understand how this flow apparatus can be regulated and controlled to yield all behaviour we observe in filopodia. 
Acknowledgements This work has been conducted as a partial result of an agreement between Faculty of Life Sciences (FLS), University of Manchester, and Brazilian National Laboratory of Scientific Computing (LNCC), in collaboration with researchers from Rio de Janeiro State University - UERJ and Federal Institute of Rio de Janeiro - IFRJ. Special thanks go to UK Biotechnology and Biological Sciences Research Council (BBSRC) for sponsoring project BB/L026724/1, to FAPERJ for project APQ-5 E-26/110.931/2014, UERJ-SR2 Visiting Researcher 2014-2016 grant, and a $\mathrm{CNPq} / \mathrm{DTI}$ grant.

The authors express their gratitude to the editors for suggestions to reformulate and deeply improve this chapter.
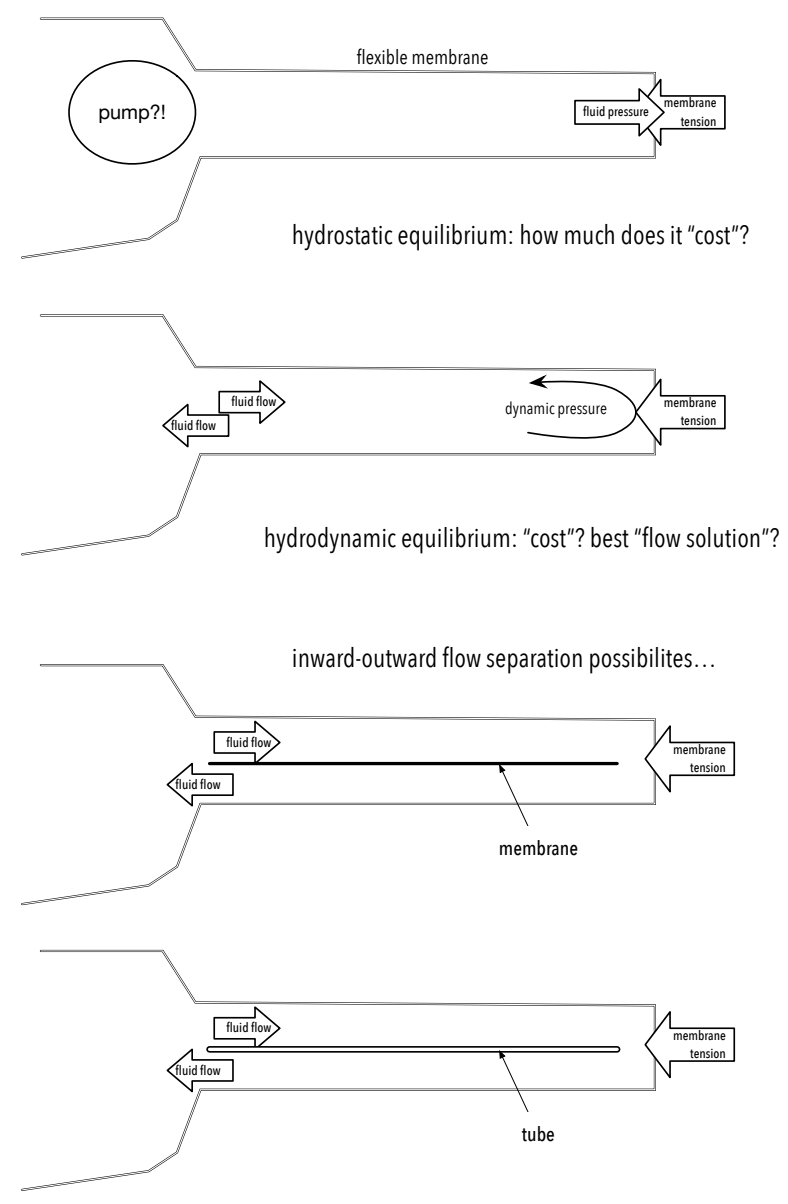

Fig. 8 Biological Quests - What is the most efficient way of sustaining the cell membrane in protrusions, and providing enough actins to keep the polymerization rate? 


\section{References}

1. Baines, A. J.: The spectrin-ankyrin-4.1-adducin membrane skeleton: adapting eukaryotic cells to the demands of animal life. Protoplasma 244: 99-131 (2010) http://www.ncbi.nlm.nih.gov/pubmed/20668894

2. Bathe, M., Heussinger, C., Claessens, M. M., Bausch, A. R. and Frey, E.: Cytoskeletal bundle mechanics. Bioph. J. 94: 2955-2964 (2008)

3. Bear, J. E. and Gertler, F. B.: ENA/VASP: towards resolving a pointed controversy at the barbed end. J. Cell Sci. 122, 1947-53 (2009) -

http://www.ncbi.nlm.nih.gov/entrez/query.fcgi?cmd=Retrieve\&db=PubMed\&dopt= Citation\&list_uids $=19494122$

4. Bindschadler, M., Osborn, E. A. and McGrath, J. L.: A mechanistic model of the actin cycle. Biophys. J. 86: 2720-2739 (2004)

5. Birbach, A.: Profilin, a multi-modal regulator of neuronal plasticity. Bioessays 30: 994-1002 (2008) http://www.ncbi.nlm.nih.gov/entrez/query.fcgi?cmd=Retrieve\&db=PubMed $\&$ dopt $=$ Citation\&list_uids $=18798527$

6. Blagoev, K. B., Shukla, K. and Levine, H.: We need theoretical physics approaches to study living systems. Phys Biol 10: 040201 (2013) http://www.ncbi.nlm.nih.gov/pubmed/23883648

7. Blanchoin, L., Boujemaa-Paterski, R., Sykes, C.and Plastino, J.: Actin dynamics, architecture, and mechanics in cell motility. Physiol Rev 94: 235-63 (2014) http://www.ncbi.nlm.nih.gov/pubmed/24382887

8. Breitsprecher, D. and Goode, B. L.: Formins at a glance. J Cell Sci 126: 1-7 (2013) http://www.ncbi.nlm.nih.gov/pubmed/23516326, http://jcs.biologists.org/content /126/1/1.full.pdf+html

9. Carlsson, A. E.: Actin dynamics: from nanoscale to microscale. Ann. Rev. Bioph. 39: 91 (2010)

10. Chesarone, M. A. and Goode, B. L.: Actin nucleation and elongation factors: mechanisms and interplay. Curr. Opin. Cell Biology 21: 28-37 (2009)

11. Cohen, J. E.: Mathematics is biology's next microscope, only better; biology is mathematics' next physics, only better. PLoS Biol 2, e439 http://www.ncbi.nlm.nih.gov/pubmed/15597117 (2004)

12. Dawes, A. T., Ermentrout, G. B., Cytrynbaum, E. N. and Edelstein-Keshet, L.: Actin filament branching and protrusion velocity in a simple 1D model of a motile cell. J. Theor. Biol. 242: 265-279 (2006)

13. Demirdzic, I. and Peric, M.: Finite volume method for prediction of fluid flow in arbitrarily shaped domains with moving boundaries. Int. J. Num. Meth. Fluids 10: 771-790 (1990)

14. Dent, E. W., Gupton, S. L. and Gertler, F. B.: The growth cone cytoskeleton in axon outgrowth and guidance. Cold Spring Harb. Perspect. Biol. 3: a001800 (2011) http://www.ncbi.nlm.nih.gov/entrez/query.fcgi?cmd=Retrieve $\& \mathrm{db}=$ PubMed\&dopt= Citation\&list_uids $=21106647$

15. Ditlev, J.A., Mayer, B.J. and Loew, L.M.: There is more than one way to model an elephant. Experiment-driven modeling of the actin cytoskeleton. Biophys. J. 104: 520-532 (2013)

16. Edwards, M., Zwolak, A., Schafer, D. A., Sept, D., Dominguez, R. and Cooper, J. A.: Capping protein regulators fine-tune actin assembly dynamics. Nat Rev Mol Cell Biol 15: 677689 (2014) - http://dx.doi.org/10.1038/nrm3869

17. Eltsov, M., Dube, N., Yu, Z., Pasakarnis, L., Haselmann-Weiss, U., Brunner, D. and Frangakis, A. S.: Quantitative analysis of cytoskeletal reorganization during epithelial tissue sealing by large-volume electron tomography. Nat. Cell. Biol. 17: 605-14 (2015) http://www.ncbi.nlm.nih.gov/pubmed/25893916

18. Erban, R., Flegg, M. B. and Papoian, G. A.: Multiscale stochastic reaction-diffusion modeling: application to actin dynamics in filopodia. Bull Math Biol 76: 799-818 (2014) http://www.ncbi.nlm.nih.gov/pubmed/23640574 
19. Evans, M. and Harlow, F. H.: The Particle-in-Cell Method for Hydrodynamic Calculations. Los Alamos Scientific Laboratory, LA-2139, 73p, New Mexico (1957)

20. Faix, J., Breitsprecher, D., Stradal, T. E. B. and Rottner, K.: Filopodia: Complex models for simple rods. Int J Biochem Cell Biol. (2009) - doi:10.1016/j.biocel.2009.02.012

21. Fletcher, D. A. and Mullins, R. D.: Cell mechanics and the cytoskeleton. Nature 463: 485-92 (2010) - http://www.ncbi.nlm.nih.gov/pubmed/20110992

22. Fujiwara, I., Vavylonis, D. and Pollard, T. D.: Polymerization kinetics of ADP- and ADP-Piactin determined by fluorescence microscopy. Proc. Natl. Acad. Sci. USA 104: 8827-8832 (2007) - http://www.ncbi.nlm.nih.gov/pubmed/17517656

23. Gallo, G.: Mechanisms underlying the initiation and dynamics of neuronal filopodia: from neurite formation to synaptogenesis. Int Rev Cell Mol Biol 301: 95-156 (2013) http://www.ncbi.nlm.nih.gov/pubmed/23317818

24. Gomez, T. M. and Letourneau, P. C.: Actin dynamics in growth cone motility and navigation. J Neurochem (2013) - http://www.ncbi.nlm.nih.gov/pubmed/24164353 (2013)

25. Gonçalves-Pimentel, C., Gombos, R., Mihály, J., Sánchez-Soriano, N. and Prokop, A.: Dissecting regulatory networks of filopodia formation in a Drosophila growth cone model. PLoS ONE 6: e18340 (2011) http://www.plosone.org/article/info\%3Adoi\%2F10.1371\%2Fjournal.pone.0018340

26. Gunawardena, J.: Beware the tail that wags the dog: informal and formal models in biology. Mol Biol Cell 25: 3441-4 (2014) - http://www.ncbi.nlm.nih.gov/pubmed/25368417

27. Gunawardena, J.: Models in biology: 'accurate descriptions of our pathetic thinking'. BMC Biology 12: 29 (2014) - http://www.biomedcentral.com/1741-7007/12/29

28. Islam, M. A.: Einstein-Smoluchowski diffusion equation: a discussion. Physica Scripta, 70: $120(2004)$

29. Kucik, D. F., Elson, E. L. and Sheetz, M. P.: Forward Transport of Glycoproteins on Leading Lamellipodia in Locomoting Cells. Nature 340: 315-317 (1989)

30. Lan, Y. and Papoian, G. A.: The stochastic dynamics of filopodial growth. Biophys J 94:3839-3852 (2008)

31. Liu, Chong and Zhigang, L.: On the Validity of the Navier-Stokes Equations for Nanoscale Liquid Flows: the Role of Channel Size. AIP Advances 1: 032108 (2011) doi:10.1063/1.3621858

32. Masuda, M. and Mochizuki, N.: Structural characteristics of BAR domain superfamily to sculpt the membrane. Semin Cell Dev Biol 21: 391-8 (2010) http://www.ncbi.nlm.nih.gov/pubmed/20083215

33. Mattila, P. K. and Lappalainen, P.: Filopodia: molecular architecture and cellular functions. Nat Rev Mol Cell Biol 9: 446-54 (2008) - http://www.ncbi.nlm.nih.gov/entrez/ query.fcgi?cmd=Retrieve $\& d b=P u b M e d \& d o p t=C i t a t i o n \& l i s t \_u i d s=18464790$

34. McGrath, J. L., Tardy, Y., Dewey, C. F., Meister, J. J. and Hartwig, J. H.: Simultaneous measurements of actin filament turnover, filament fraction, and monomer diffusion in endothelial cells. Biophys. J. 75: 2070-2078 (1998)

35. Mellor, H.: The role of formins in filopodia formation. Biochim Biophys Acta (2009) doi:10.1016/j.bbamcr.2008.12.018 - http://www.ncbi.nlm.nih.gov/entrez/ query.fcgi?cmd=Retrieve $\& \mathrm{db}=$ PubMed\&dopt=Citation\&list_uids $=19171166$

36. Mogilner, A., Allard, J. and Wollman, R.: Cell polarity: quantitative modeling as a tool in cell biology. Science 336: 175-179 (2012) http://www.ncbi.nlm.nih.gov/pubmed/22499937

37. Mogilner, A. and Oster, G.: Cell motility driven by actin polymerization. Bioph. J. 71: 30303045 (1996) - http://www.ncbi.nlm.nih.gov/pubmed/8968574

38. Mogilner, A. and Rubinstein, B.: The physics of filopodial protrusion. Bioph. J. 89: 782-95 (2005) - http://www.ncbi.nlm.nih.gov/pubmed/15879474

39. Mogilner, A., and Edelstein-Keshet, L.: Regulation of actin dynamics in rapidly moving cells: a quantitative analysis. Bioph. J. 83: 1237-1258 (2002)

40. Mullins, R. D. and Hansen, S. D.: In vitro studies of actin filament and network dynamics. Curr Opin Cell Biol 25: 6-13 (2013) -

http://www.sciencedirect.com/science/article/pii/S0955067412001901 
41. Oosawa, F., Asakura, S. and Ooi, T.: G-F transformation of actin as a fibrous condensation. J. Polym. Sci. 37: 323-336 (1959) - http://dx.doi.org/10.1002/pol.1959.1203713202

42. Oster, G. and Perelson, A. S.: Cell protrusions. In: Frontiers in mathematical biology. Springer, Berlin-Heidelberg, 53-78 (1994)

43. Othmer, H.: Actin Cytoskeleton, Multi-scale Modeling. In: Encyclopedia of Applied and Computational Mathematics (B. Engquist, Ed.), 17-23. Springer Berlin-Heidelberg (2015) - http://dx.doi.org/10.1007/978-3-540-70529-1_60

44. Pak, C. W., Flynn, K. C. and Bamburg, J. R.: Actin-binding proteins take the reins in growth cones. Nat Rev Neurosc. 9: 136-47 (2008) http://www.ncbi.nlm.nih.gov/entrez/query.fcgi?cmd=Retrieve \&db=PubMed \&dopt= Citation\&list_uids $=18209731$

45. Perelson, A. S. and Coutsias, E. A.: A moving boundary model of acrosomal elongation. J. Math. Biol. 23: 361-379 (1986)

46. Peskin, C. S., Odell, G. M. and Oster, G. F.: Cellular motions and thermal fluctuations: the Brownian ratchet. Bioph. J. 65: 316 (1993)

47. Pollard, T. D.: Rate constants for the reactions of ATP-and ADP-actin with the ends of actin filaments. J. Cell Biol. 103: 2747-2754 (1986)

48. Pollard, T. D.: Regulation of actin filament assembly by Arp $2 / 3$ complex and formins. Annu. Rev. Biophys. Biomol. Struct. 36: 451-477 ( 2007)

49. Pollard, T. D. and Cooper, J. A.: Actin and actin-binding proteins. A critical evaluation of mechanisms and functions. Ann Rev Biochem, 55: 987-1035 (1986)

50. Potter, D.: Computational Physics. John Wiley \& Sons, Chichester(1973)

51. Prokop, A., Beaven, R., Qu, Y. and Sánchez-Soriano, N.: Using fly genetics to dissect the cytoskeletal machinery of neurons during axonal growth and maintenance. J. Cell Sci. 126: 2331-41 (2013) - http://dx.doi.org/10.1242/jcs.126912

52. Prokop, A., Küppers-Munther, B. and Sánchez-Soriano, N.: Using primary neuron cultures of Drosophila to analyse neuronal circuit formation and function. In The making and un-making of neuronal circuits in Drosophila (B. A. Hassan, Ed.), Vol. 69: 225-247. Humana Press, New York (2012) -

http://www.springerlink.com/content/t07618161235u475/\#section=1102403\&page =1 http://dx.doi.org/10.1007/978-1-61779-830-6_10

53. Prokop, A., Sánchez-Soriano, N., Gonçalves-Pimentel, C., Molnár, I., Kalmr, T. and Mihály, J.: DAAM family members leading a novel path into formin research. Commun Integr Biol 4: 538-42 (2011) - http://www.landesbioscience.com/journals/cib/article/16511/

54. Pronk, S., Geissler, P. L. and Fletcher, D. 1. A.: Limits of filopodium stability. Phys. Rev. Let. 100: 258102 (2008)

55. Rao, Y. and Haucke, V.: Membrane shaping by the Bin/amphiphysin/Rvs (BAR) domain protein superfamily. Cell Mol Life Sci 68: 3983-93 (2011) http://www.ncbi.nlm.nih.gov/pubmed/21769645

56. Safari, F. and Suetsugu, S.: The BAR Domain Superfamily Proteins from Subcellular Structures to Human Diseases. Membranes (Basel) 2: 91-117 (2010) http://www.ncbi.nlm.nih.gov/pubmed/24957964

57. Sánchez-Soriano, N., Gonçalves-Pimentel, C., Beaven, R., Haessler, U., Ofner, L., Ballestrem, C. and Prokop, A.: Drosophila growth cones: a genetically tractable platform for the analysis of axonal growth dynamics. Dev. Neurobiol. 70: 58-71 (2010) http://www3.interscience.wiley.com/cgi-bin/fulltext/123188795/PDFSTART http://www.ncbi.nlm.nih.gov/entrez/query.fcgi?cmd=Retrieve\&db=PubMed\&dopt= Citation\&list_uids $=19937774$

58. Sánchez-Soriano, N., Tear, G., Whitington, P. and Prokop, A.: Drosophila as a genetic and cellular model for studies on axonal growth. Neural Develop. 2: 9 (2007) http://www.ncbi.nlm.nih.gov/entrez/query.fcgi? $\mathrm{cmd}=$ Retrieve $\& \mathrm{db}=\mathrm{PubMed} \& \mathrm{dopt}=$ Citation\&list_uids $=17475018$

59. Schmid, M. F., Sherman, M. B., Matsudaira, P. and Chiu, W.: Structure of the acrosomal bundle. Nature 431: 104-107 (2004) 
60. Vavylonis, D., Yang, Q. and O'Shaughnessy, B.: Actin polymerization kinetics, cap structure, and fluctuations. Proc. Natl. Acad. Sci. USA 102: 8543-8548 (2005) -

http://www.ncbi.nlm.nih.gov/pubmed/15939882

61. Wegner, A.: Head to tail polymerization of actin. J. Mol. Biol. 108: 139-150 (1976) http://www.ncbi.nlm.nih.gov/pubmed/1003481

http://www.sciencedirect.com/science/article/pii/S0022283676801003

62. Wilson, K. G.: Grand challenges to computational science. Future Generation Computer Systems 5: 171-189 (1989). doi: 10.1016/0167-739X(89)90038-1

63. Zicha, D., Dobbie, I. M., Holt, M. R., Monypenny, J., Soong, D. Y., Gray, C. and Dunn, G. A.: Rapid actin transport during cell protrusion. Science 300: 142-145 (2003)

64. Zhuravlev, P. I., Der, B. S. and Papoian, G. A.: Design of active transport must be highly intricate: a possible role of myosin and Ena/VASP for G-actin transport in filopodia. Bioph. J. 98: 1439-1448 (2010)

65. Zhuravlev, P. I. and Papoian, G. A.: Protein fluxes along the filopodium as a framework for understanding the growth-retraction dynamics: the interplay between diffusion and active transport. Cell Adhesion \& Migration 5: 448-456 (2011) 